\title{
A DE RHAM TYPE THEOREM FOR ORBIT SPACES
}

\author{
ANDREI VERONA
}

(Communicated by Haynes R. Miller)

\begin{abstract}
Let $G$ be a compact Lie group and $M$ be a smooth $G$-space. We prove that the real cohomology algebra of the orbit space $M / G$ is isomorphic to the homology algebra of the de Rham complex of $G$-basic differential forms on $M$.
\end{abstract}

Let $G$ be a compact Lie group and $M$ be a smooth $G$-manifold, i.e. $M$ is a paracompact, $C^{\infty}$-manifold and there is given a $C^{\infty}$-map $f: G \times M \rightarrow M, f(g, x)=$ $g x$, such that (i) $g(h x)=(g h) x$ for $g, h \in G, x \in M$; and (ii) $e x=x$ for $x \in M$, $e \in G$ being the neutral element (see [2] for all other notions and results concerning $G$-manifolds that will be used in this note). For $g \in G$ let $L_{g}: M \rightarrow M$ denote the diffeomorphism $L_{g}(x)=g x$. For $x \in M$ let $G_{x}=\{g \in G ; g x=x\}$ be the isotropy subgroup at $x$ and $G x=\{g x ; g \in G\}$ be the $G$-orbit through $x$. Let $M / G$ denote the orbit space and $p: M \rightarrow M / G$ be the canonical projection.

Let $\operatorname{Lie}(G)$ be the Lie algebra of $G$ and for $X \in \operatorname{Lie}(G)$ let $X^{*}$ be the induced vector field on $M$ : for each $x \in M, X^{*}(x)$ is the vector tangent to the curve $t \mapsto(\exp (t X)) x$ at $t=0$.

For $r \geq 0$ let $\mathscr{D}^{r}(M)$ denote the (real) vector space of $C^{\infty}$-forms on $M$ of degree $r$. Given $X \in \operatorname{Lie}(G)$ let $i_{X}: \mathscr{D}^{r}(M) \rightarrow \mathscr{D}^{r-1}(M)$ be the interior product with $X^{*}$. Let also $d: \mathscr{D}^{r}(M) \rightarrow \mathscr{D}^{r+1}(M)$ denote the exterior derivative. A form $\omega \in \mathscr{D}^{r}(M)$ is called $G$-basic if

$$
\left(L_{g}\right)^{*}(\omega)=\omega \quad \text { and } \quad i_{X}(\omega)=0 \quad \text { for any } g \in G, X \in \operatorname{Lie}(G) .
$$

We shall denote by $\mathscr{D}_{G}^{r}(M)$ the real vector space of $G$-basic forms on $M$ of degree $r$. It is easy to check that $d\left(\mathscr{D}_{G}^{r}(M)\right) \subset \mathscr{D}_{G}^{r+1}(M)$ and therefore we can consider the quotient $H_{G}^{r}(M)=\left(\operatorname{ker}(d) \cap \mathscr{D}_{G}^{r}(M)\right) / d\left(\mathscr{D}_{G}^{r-1}(M)\right)$. Let $H_{G}^{*}=\bigoplus_{r>0} H^{r}(M)$ and notice that the exterior product determines an algebra structure on $H_{G}^{*}(M)$.

THEOREM. The algebra $H_{G}^{*}(M)$ is isomorphic to the real cohomology algebra of $M / G$.

PROOF. For $U \subset M / G$ open and $r \geq 0$, let $S^{r}(U)=\mathscr{D}_{G}^{r}\left(p^{-1}(U)\right)$. If $V \subset U$ is also open, there exists an obvious restriction homomorphism $S^{r}(U) \rightarrow S^{r}(V)$. The assignment $U \mapsto S^{r}(U)$ is a sheaf over $M / G$; denote it $\mathbf{S}^{r}$. The exterior derivative gives rise to sheaf morphisms $d: \mathbf{S}^{r} \rightarrow \mathbf{S}^{r+1}, r \geq 0$. The Theorem will follow if we can prove that

$$
0 \rightarrow \mathbf{R} \rightarrow \mathbf{S}^{0} \rightarrow \mathbf{S}^{1} \rightarrow \mathbf{S}^{2} \rightarrow \cdots
$$

Received by the editors April 1, 1987 and, in revised form, August 20, 1987. 1980 Mathematics Subject Classification (1985 Revision). Primary 57S15, 58A12. Research partially supported by NSF Grant DMS-8509186. 
is a fine resolution of $\mathbf{R}$, the constant sheaf over $M / G$ with stalk $R$ (the reals). Since there are partitions of unity on $M$ consisting of $G$-invariant $C^{\infty}$-functions, $\mathbf{S}^{0}$ is fine and therefore so is each $\mathbf{S}^{r}, r \geq 1$.

Let $x \in M, H=G_{x}$, and $N=T_{x} M / T_{x}(G x)$ be the normal space at $x$ to the orbit $G x$. The action of $G$ on $M$ induces a linear action of $H$ on $N$ (the so-called slice (or isotropy) representation), and there exists an open, $G$-invariant neighborhood $U$ of $x$ in $M$ which is $G$-equivariantly diffeomorphic to the twisted product $G \times{ }_{H} N$ on which $G$ acts by left multiplication (this is the "slice theorem"; recall that $G \times{ }_{H} N$ is the orbit space $(G \times N) / H$, where $H$ acts on $G \times N$ by $\left.h(g, v)=\left(g h^{-1}, h v\right)\right)$. Consider the commutative diagram

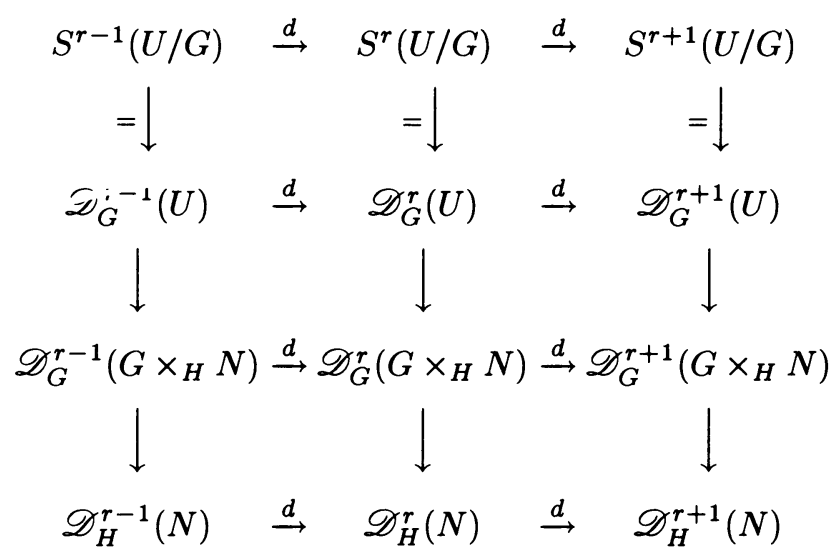

in which the second row of vertical maps is induced by the above-mentioned diffeomorphism and the third one by the map $x \mapsto[e, x]: N \rightarrow G \times_{H} N$ (here $[e, x]$ denotes the $H$-orbit through $(e, x) \in G \times N)$. It is easy to see that all vertical maps are isomorphisms. Let us check that the bottom row is exact. To this end consider the homotopy operator $A: \mathscr{D}^{r}(N) \rightarrow \mathscr{D}^{r-1}(N)$ (see, for example, [4, p. 128]). A direct computation shows that for $\omega \in \mathscr{D}^{r}(N), h \in H$ and $X \in \operatorname{Lie}(H)$

$$
A\left(\left(L_{h}\right)^{*}(\omega)\right)=\left(L_{h}\right)^{*}(A(\omega)) \quad \text { and } \quad A\left(i_{X}(\omega)\right)=i_{X}(A(\omega))
$$

(the first equality is true for any $C^{\infty}$-map $f: N \rightarrow N$ such that $f(t x)=t f(x)$, $t \in R, x \in N$; the second equality is true for any vector field $Y$ on $N$ such that $Y(t x)=t Y(x), t \in R, x \in N$, where $T_{t x}(N)$ and $T_{x}(N)$ are identified as usually to $N)$.

It follows that $A\left(\mathscr{D}_{H}^{r}(N)\right) \subset \mathscr{D}_{H}^{r-1}(N)$. Thus if $\omega \in \mathscr{D}_{H}^{r}(N)$ is such that $d \omega=0$, then $\omega=d \theta$ with $\theta=A \omega \in \mathscr{D}_{H}^{r-1}(N)$. This proves the exactity of the bottow row. Therefore, the first row is also exact.

Since the open sets of the form $U / G$ with $U$ as above form a fundamental system of neighborhoods of $p(x)$ in $M / G$, it follows that $(*)$ is a resolution of $\mathbf{R}$. The Theorem follows from Theorem 4.7.1 in [3].

Note. The particular case of the Theorem when all the isotropy groups are finite was proved in [1, Lemma 10.2$]$ by other methods. 


\section{REFERENCES}

1. M. F. Atiyah, Elliptic operators and compact groups, Lecture Notes in Math., vol. 401, SpringerVerlag, Berlin, Heidelberg and New York, 1974.

2. G. Bredon, Introduction to compact transformation groups, Academic Press, New York and London, 1972

3. R. Godement, Théorie des faisceaux, Hermann, Paris, 1964.

4. R. Narasimhan, Analysis on real and complex manifolds, Masson, Paris; North-Holland, Amsterdam; Amsterdam Elsevier, New York, 1973.

Department of Mathematics, California State University, los Angeles, CALIFORNIA 90032 